\title{
The scale of heart failure: diagnosis and management issues for primary care
}

\author{
F D R Hobbs
}

Heart failure is a significant public health issue. Epidemiological surveys using clinical findings suggest that between $1-2 \%$ of western adult populations are affected by heart failure. More recent data, however, based on objective cardiac assessment, suggest that $2 \%$ is the more accurate figure. ${ }^{12}$

Further evidence from the US show that in the last 20 years there has been a fourfold increase in unadjusted mortality rates for heart failure (fig 1). The most obvious reason for this is the increase in the aging population, although changes in classification may be another factor. Prevalence is also increasing because more people are surviving myocardial infarcts (fig 2). There is an inexorable relation between patients surviving an acute myocardial infarction and the subsequent development of heart failure. Therefore, if the number of patients surviving acute myocardial infarction increase, it is almost certain that an increasing heart failure prevalence will follow.

\section{Impact of heart failure}

There are three issues to consider in terms of the impact of heart failure. Mortality is related

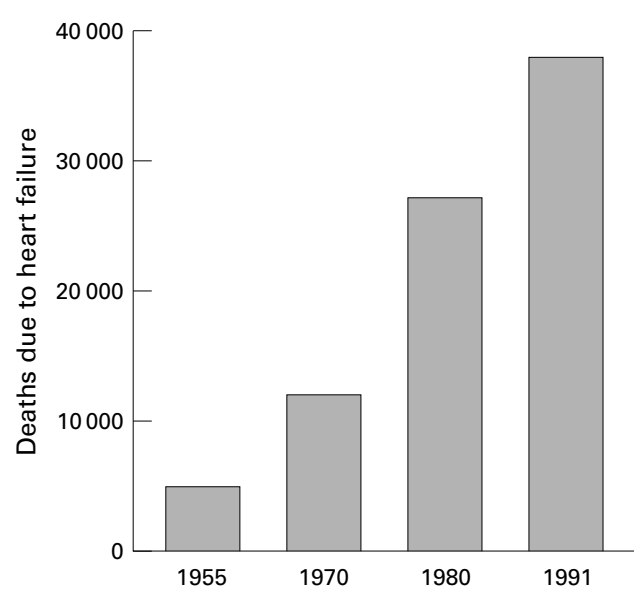

Figure 1 Increasing prevalence of heart failure.

Head of Division of Primary Care, Public and Occupational Health, University of Birmingham, Birmingham B15 2TT, UK

F D R Hobbs

Correspondence to: Professor Hobbs
Figure 2 Risk of heart failure following myocardial infarction. to disease severity, so prognosis is determined by stage of heart failure. Looking at heart failure overall, the five year mortality rate of $50 \%$ is analogous to that of many cancers. The prognosis for moderate and severe heart failure is almost identical to colorectal cancer ${ }^{3}$ and worse than breast ${ }^{4}$ or prostatic cancer, ${ }^{5}$ which develop at a similar age to heart failure (table 1). Interestingly, the impact of breast cancer is considered sufficiently significant to warrant a national screening programme for women from the age of 50 years. In the US there are also formal screening programmes for prostatic cancer.

The second issue relates to high health care costs, largely related to the use of hospital beds. Heart failure is an expensive condition to treat, largely because it results in high rates of admission. ${ }^{6}$ It is the single most common cause of admissions in the US and the UK, and therefore has a very high impact in terms of health care costs.

The third issue that relates to the impact of heart failure is quality of life. Data from the echocardiographic heart of England screening (EcHoES) study illustrate this. ${ }^{7}$ The study, similar to Glasgow's monitoring trends and determinants in cardiovascular disease (MONICA) heart scan survey, screened a randomly selected adult population and calculated their quality of life score. A randomly selected population of patients with a pre-existing label of heart failure was also screened. Quality of life scores were determined using short form 36 (SF36), and compared the measure between a population of patients who have definite heart failure standardised against the randomly selected general population with no heart failure. SF36 is probably the most widely used quality of life score, which has the advantage of being self administered, quick to complete, and repeatable. There are eight modalities: physical functioning, role physical, role mental, social functioning, mental health, energy, pain, and an overall perception of health.

All stage heart failure has a very substantial and significant effect upon quality of life, particularly in terms of physical functioning and overall perception of health. So there are

Table 1 Survival rates compared with heart failure ${ }^{3-5}$

\begin{tabular}{llll}
\hline & \multicolumn{3}{l}{ Survival rates (\%) } \\
\cline { 2 - 4 } & 1 year & 2 years & 3 years \\
\hline Breast cancer & 88 & 80 & 72 \\
Prostate cancer & 75 & 64 & 55 \\
Colon cancer & 56 & 48 & 42 \\
Heart failure & 67 & 41 & 24 \\
\hline
\end{tabular}


highly significant and substantial differences in quality of life between the general and heart failure populations. Quality of life varies widely according to severity. In class I, patients are asymptomatic, enjoying a quality of life almost identical to that of the general population. Quality of life deteriorates progressively with worsening heart failure.

\section{Diagnostic accuracy}

The accuracy of heart failure diagnosis is questionable. Several studies suggest that when patients labelled as having heart failure are screened using objective assessment criteria, only around one third are found to have true heart failure. ${ }^{89}$ Of the population of 600 randomly selected patients with a label of heart failure in the EcHoES study, only $25 \%$ had definite left ventricular dysfunction, although an additional $12 \%$ were borderline.${ }^{10}$ However, $23 \%$ of patients in the EcHoES study labelled as having heart failure were in atrial fibrillation and, of these, nearly $60 \%$ had a normal left ventricular function. A further $20 \%$ had valvar abnormalities. So in the EcHoES study around $60 \%$ of patients showed evidence of structural abnormalities.

European working party guidelines recommend that heart failure is diagnosed on the basis of appropriate symptoms, plus objective evidence of structural cardiac abnormality and, ideally, a response to treatment. ${ }^{11}$ By these standards, approximately $60 \%$ of patients in the EcHoES study with a label of heart failure would meet the criteria in terms of having some structural abnormality on objective cardiac testing, although only a minority of patients would have left ventricular dysfunction. It is important to identify left ventricular dysfunction in order to assess patients' suitability for angiotensin converting enzyme (ACE) inhibitor treatment. However, there are other conditions for which appropriate treatment is likely to improve cardiac function. The most notable is atrial fibrillation where rhythm control may also improve the symptoms which lead patients to be labelled as suffering from heart failure.

\section{Improving care}

Given that heart failure is going to have an increasing impact on both patients and health care systems, it is reasonable to expect concerted efforts to improve care, particularly since there are evidence based treatments available to improve both prognosis and quality of life. Unfortunately repeated data show that physicians do not act on this evidence in terms of clinical practice; only a minority of patients who have heart failure - around a third in most surveys-are taking ACE inhibitors, the treatments supported by a substantial evidence base in terms of improving mortality and morbidity. ${ }^{12}$

This is not just an issue for general practice but, to a lesser degree, also for hospital practice. Where you might see only a third of heart failure patients in primary care on ACE inhibitors, in hospitals this figure is still only likely to reach about two thirds. ${ }^{13}$
The other issue is not just underuse but undertreatment in terms of failing to use target doses recommended on the basis of clinical trials. Generally, there is improvement with regard to physician uptake of evidence concerning use of cardiovascular drugs. Aspirin and $\beta$ blocker use after myocardial infarction has improved considerably in recent years, both in primary and secondary care practice.

However, 1998 data from hospital practice in the US show that where enalapril is prescribed in heart failure, $80 \%$ of patients are being dosed with $<15 \mathrm{mg}$ - a dose considered inadequate on the basis of heart failure trials.

\section{Reasons for underperformance in heart failure management}

To determine the reasons behind underuse and undertreatment in the UK, Houghton looked at a relatively small population of general practitioners (GPs) around Nottingham. ${ }^{14} \mathrm{He}$ found that ACE inhibitors were underprescribed, not because the benefits were misunderstood-indeed, in that small qualitative study most GPs were aware of the benefits of ACE inhibitor treatment-but because the GPs were worried about adverse effects. Since objective evidence is required to diagnose heart failure, there are practical difficulties in diagnoses in primary care. Euro-heart failure (Euro-HF), a large pan-European study conducted last year, surveyed a random selection of GPs across six European countries. ${ }^{15}$ They were asked a series of questions about their perceptions of heart failure, including how they reached a diagnosis. In most countries, GPs were not ordering echocardiograms to assist their diagnosis of heart failure. In the UK it was being used in about one third of patients and only a small proportion of doctors had open access to echocardiography. So the most reliable assessment of heart failure, in terms of ventricular function, was largely not available to most GPs.

While GPs can perform ECGs, one of the problems with electrocardiography in primary care is that expert opinion is still required for interpretation. Generalists in primary care who perhaps review only one or two ECGs per week are going to miss many minor changes that would be detected by a specialist. Issues of access to investigation therefore influence the ability of primary care physicians to diagnose accurately.

\section{Perceptions of risk}

The Euro-HF study also asked physicians how they perceived particular treatments-in this instance ACE inhibitors - with regard to symptom relief, side effects, evidence of reduced mortality, and risks. ${ }^{15}$ The striking finding to emerge was that most doctors in Europe, perhaps with the notable exception of Spain, were aware of the evidence of reduced mortality accompanying the use of ACE inhibitors. Their underuse, which they acknowledged, was because they perceive significant risks associated with use of ACE inhibitors. In the UK, compared to continental Europe, there are significantly more concerns 
over the risks associated with ACE inhibitor use in heart failure. GPs in the UK worried about old fears which were of concern during the introduction of ACE inhibitors-that is, perceptions of renal impairment and hypotension. Neither concern should provide impediment to the use of ACE inhibition today. All that is needed is to monitor creatinine and adjust the dose if necessary. Dosing can be increased gradually and the first dose can be administered at night to avoid hypotension. ACE inhibitors were introduced in the UK in such a way that for the first 18 months their use was initiated only in hospitals. Unfortunately, this is precisely the way $\beta$ blockers are now being introduced in heart failure. Once again concerns are being raised about a potentially sound treatment and the implications, unless we are careful, are going to be much the same as those surrounding the uptake of $\mathrm{ACE}$ inhibitors in primary care practice.

\section{Conclusions}

Significant issues relating to under- and misdiagnosis of heart failure across Europe are indisputable. Primary care physicians are almost universally diagnosing on clinical grounds because they have only limited access to objective testing. In terms of treatment, most physicians claim to use ACE inhibitors but they do so only in a proportion of patients and in lower than recommended doses. The reasons for this are not because treatment benefits are being misunderstood but are almost certainly related to perceptions of $\mathrm{ACE}$ inhibitor risks and adverse effects.

Education and feedback can alter physician performance. In the EcHoES study, having identified patients with left ventricular dysfunction, letters were sent to their GPs telling them that their patients had this condition and recommending ACE inhibitor treatment. This resulted in a significant uptake of ACE inhibitor treatment in most practices.

Concerns can be overcome with quite simple definitive recommendations confirming the diagnosis and recommending treatments about which they might otherwise have felt concern.

In terms of more recent treatments, $\beta$ blockers in particular, there is a major problem. Primary care physicians in the UK are certain to consider $\beta$ blockers positively contraindicated in heart failure. Even the British National Formulary is currently ambivalent on the subject; this will have to be changed before primary care physicians will consider changing their practice.

If heart failure was regarded as a type of cancer of the heart, we would treat it very differently. Patients who present with cancer generally feel relatively well. Their cancer is very rapidly investigated and staged; they then undergo treatments which often show very little survival gain but nonetheless make patients feel ill. So they proceed from feeling well to feeling ill in the hope of increased survival. Patients with heart failure, on the other hand, present feeling ill yet are missing treatments which will improve mortality and make them feel better.
The forthcoming UK national service framework for coronary heart disease is going to confront primary care head on where these issues are concerned because it will become more difficult not to act on scientific evidence. These are the sorts of issues that we need to consider if we are to start educating primary care physicians about evidence based treatments. Providing the evidence and the rationale is not enough. There needs to be assistance in case selection and in grading patients according to heart failure stage.

If patients are going onto $\beta$ blocker treatment, they are going to be started in hospital. If patients are subsequently discharged to physicians who believe $\beta$ blockers are contraindicated in heart failure, there may be problems with treatment being stopped. The point has been made that patients on $\beta$ blockers may temporarily feel worse before feeling better, and therefore it is crucial for patients to understand that so as not to stop their treatment themselves.

Whether $\beta$ blockade for heart failure should be prescribed in primary care at the present time is debatable. There is currently only one licensed product available for heart failure and that is restricted to initiation and up titration in hospital. However, it should not be long before there are sufficient data to support the use of $\beta$ blocker treatment for heart failure in primary care. The important thing will be to reassure patients that it is going to take a while for them to feel better. In primary care, where it is easy to see patients regularly, advice will probably be to start low and increase very slowly, but as yet we do not have sufficient evidence for this.

1 Davis RC, Hobbs FDR, McLeod S, et al. Prevalence of left ventricular dysfunction and valve disease in a typical English region [abstract]. $\mathcal{F}$ Am Coll Cardiol 1998; 33(suppl 1):82.

2 McDonagh TA, Morrison CE, Lawrence A, et al. Symptomatic and asymptomatic left-ventricular systolic dysfunction in an urban population. Lancet 1997;350:829-33.

3 Joint Report of the West Midlands Director of Public Health and the West Midlands Regional Cancer Registry. Cancer and health. Birmingham: WMRHA, 1995:210.

4 Joint Report of the West Midlands Director of Public Health and the West Midlands Regional Cancer Registry. Cancer and the West Midlands Regional Cancer Re

5 Joint Report of the West Midlands Director of Public Health and the West Midlands Regional Cancer Registry. Cancer and health. Birmingham: WMRHA, 1995:82.

6 McMurray J, McDonagh T, Morrison CE, et al. Trends in hospitalisation for heart failure in Scotland 1980-1990. Eur Heart f 1993;14:1158-62.

7 Davis R, Hobbs FDR, Kenkre JE, et al. Quality of life in heart failure, as measured by SF-36 health status questionnaire [abstract]. Eur Heart f 1998;19(suppl):S639.

8 Weeldon NM, McDonald TM, Fluker CJ, et al. Echocardiography in chronic heart failure in the community. QfM 1993;86:17-20

9 Remes J, Miettinen H, Reunanen A, et al. Validity of clinical diagnosis of heart failure in primary health care. Eur Heart 1991;12:315-21.

10 Hobbs FDR, Davis RC, McLeod S, et al. Prevalence of heart failure in 'high risk' groups [abstract]. $\mathcal{f}$ Am Coll Cardiol 1998;31 (suppl 5):85C.

11 The Task Force on Heart Failure of the European Society of Cardiology. Guidelines for the diagnosis of heart failure. Eur Heart $\mathcal{F}$ 1995;16:741-51.

2 SOLVD Investigators. Effect of enalapril on survival in patients with reduced left ventricular ejection fraction and congestive heart failure. N Engl F Med 1991;325:293-302.

13 Anon. Failure to treat heart failure [editorial]. Lancet 1992; 339:278-9.

14 Houghton AR, Cowley AJ. Why are angiotensin converting enzyme inhibitors underutilised in the treatment of heart
failure by general practitioners? Int f Cardiol 1997;59:7-10.

15 Hobbs FDR, Wilson S, Jones MI, et al. European survey (EuroHF) of primary care physician perceptions and practice in heart failure diagnosis and management [abstract]. Eur Heart f 1998;19(suppl): 154 . 